\title{
pesquisa
}

CAÑIZARES, K.A.L.; GOTO, R. Crescimento e produção de pepino em função da enxertia. Horticultura Brasileira, Brasília, v.16, n.2, p.110-113, novembro 1998.

\section{Crescimento e produção de híbridos de pepino em função da enxertia. ${ }^{1}$}

\author{
Kathia A.L. Cañizares; Rumy Goto. \\ UNESP - FCA - Horticultura, C. Postal 237, 18.603-970 Botucatu - SP.
}

RESUMO

O presente trabalho objetivou avaliar o efeito da enxertia no crescimento, produção e na qualidade visual dos frutos de híbridos de pepino ('Nikkey' e 'Ancor 8') em híbridos de abóbora ('Ikky' e 'Tetsukabuto'), em cultivo protegido. O ensaio foi conduzido na área experimental da FCA da UNESP - Botucatu entre 29 de março e 30 de agosto de 1996, num ambiente protegido com controle parcial das condições ambientais. Os seis tratamentos resultantes da combinação dos dois híbridos de pepino enxertados por garfagem em fenda cheia sobre os dois híbridos de abóbora além dos dois híbridos sem enxertia, foram distribuídos em delineamento de blocos ao acaso com quatro repetições, sendo as parcelas constituídas por três plantas, crescidas em vasos de plástico e preenchidos com dez litros de solo corrigido. As características avaliadas foram: altura das plantas, número de internódios/planta, peso da matéria seca da raiz, número de frutos totais e comerciáveis por $\mathrm{m}^{2} \mathrm{e}$ presença de brilho nos frutos. A enxertia influenciou no crescimento, rendimento e na qualidade dos pepinos. As plantas enxertadas apresentaram valores superiores para altura, número de internódios/planta e número de frutos totais e comerciáveis. Todos os frutos das plantas enxertadas sobre "Ikky" apresentaram brilho na casca.

\begin{abstract}
Cucumber hybrids growth and yield accordingly to grafting.

The purpose of the present research was to evaluate the effect of grafting on yield and visual fruit quality of cucumber hybrids ('Nikkey' and 'Ancor 8') grafted on squash hybrids ('Ikky' and 'Tetsukabuto'). The trial was carried out in the "FCA - UNESP Botucatu" experimental area, from March 291996 to August 30 1996, under vinyl house, with parcial environmental control. Cleft grafting was applied. Six treatments (four combinations of scion and rootstock, and two cucumber hybrids without grafting) were set out in a randomized block design, with four replications each of three plants. Plants were grown in ten liter plastic pots using corrected soil. Plant height, number of internodes, root dry matter weight, number of total and commercial fruits per $\mathrm{m}^{2}$, and fruit shine were evaluated. Grafted plants had higher growth, development, yield and fruit quality than non-grafted plants. Grafting increased plant height, number of internodes per plant, root dry weigth and number of total and commercial fruits. Fruits of cucumber plants grafted on "Ikky" were shine.
\end{abstract}

Keywords: Cucumis sativus L, Cucurbitaceae.
$\mathrm{E}$ $\mathrm{m}$ cultivo sob ambiente protegido em diversos países, o pepino constitui uma importante hortaliça, já que ocupa o segundo lugar após o tomate (Silva et al., 1995). Porém, o uso do solo de forma intensiva, praticado neste sistema de produção de hortaliças tem trazido problemas fitossanitários. Assim, no Japão, Oda et al. (1993) atribuíram $85 \%$ do fracasso da produção de hortaliças, em cultivos sucessivos sob ambiente protegido, às doenças ocasionadas por patógenos do solo. Para prevenir esta ocorrência, a enxertia tem sido usada na produção de pepino e também de melancia, melão, berinjela e tomate.

Em geral, as plantas enxertadas têm manifestado resistência a doenças, tolerância a níveis elevados de umidade e concentração de sais no solo, tolerância à alcalinidade e resistência ao frio (Janick, 1966). Epecificamente o pepino enxertado tem sua produção consideravelmente aumentada, sendo que através da enxertia podem determinados cavalos induzir ao aumento do vigor da copa (Janowski \& Skapski, 1985). O porta-enxerto Cucurbita ficifolia tem apresentado tolerância a Phomopsis sclerotioides e resistência ao Verticillium (Yamakawa, 1982). Tem sido verificada resistência a temperaturas baixas em plantas de pepino enxertadas em determinados cavalos (Liebig, 1985), fungos do solo e nematóides (Oda et al., 1993), umidade, salinidade e excesso de água (Oda, 1995). Por outro lado, os frutos de plantas enxertadas em porta-enxertos específicos perdem a cerosidade característica, ficando com brilho (Kawaide, 1985).

Considerando a importância de manter o estado fitopatológico do solo nas melhores condições possíveis, por um longo período de tempo nos ambientes protegidos, faz-se necessário considerar a enxertia como um método alternativo de produção, visando controlar em forma preventiva a ocorrência de doenças do solo, assim como amenizar o efeito dos nematóides. Com estes argumentos e com a finalidade de estabelecer as possíveis diferenças do desenvolvimento e produção entre plantas enxertadas e não enxertadas, o presente trabalho objetivou avaliar o efeito da enxertia no crescimento da planta, no rendimento $\mathrm{e}$ na qualidade visual dos frutos de híbridos de pepino enxertados em abóbora.

\footnotetext{
${ }^{1}$ Com apoio da FAPESP
} 


\section{MATERIAL E MÉTODOS}

O ensaio foi conduzido sob estrutura simples de metal, tipo arco, de $100 \mathrm{~m}^{2}$, coberto por filme plástico de 75 milimicras e com controle parcial das condições ambientais da FCA da UNESP Botucatu, entre 29 de março e 31 de agosto de 1996.

A semeadura realizou-se em bandejas de poliestireno expandido de 128 células, uma semente/célula, aos 29 de março de 1996 (abóbora) e aos 03 de abril de 1996 (pepino). Aos 09 de abril de 1996 foi feita a enxertia pelo método de garfagem em fenda cheia (Oda, 1995), destacando-se o meristema apical da abóbora, onde foi feito um corte de $1,0-1,5 \mathrm{~cm}$ em sentido longitudinal. $\mathrm{Na}$ muda de pepino realizou-se um corte em cunha $3,0 \mathrm{~cm}$ abaixo das folhas cotiledonares. $\mathrm{O}$ enxerto foi fixado por um 'clip' especial, permanecendo por quatro dias numa câmara coberta de filme de polietileno de 100 milimicras, com umidade relativa acima de $90 \%$ e aproximadamente $30^{\circ} \mathrm{C}$. A adaptação da muda culminou aos dez dias e o transplante $(0,7 \times 0,5 \mathrm{~m})$ para vasos de plástico de dez litros de capacidade ocorreu aos 23 de abril de 1996.

Os vasos foram preenchidos com dez litros de um solo corrigido previamente. Assim, retirou-se uma camada à profundidade de 0 a $20 \mathrm{~cm}$ de um solo classificado por Carvalho et al. (1983) como Latossolo vermelho - escuro, álico, de textura média, observando-se tratar de terra de reação ácida, com baixa por-

Tabela 1. Análise química ${ }^{1}$, antes e após 30 dias da correção inicial do solo utilizado para preencher os vasos. Botucatu, UNESP - FCA, 1996.

\begin{tabular}{|c|c|c|c|c|c|c|c|c|c|c|}
\hline & \multirow{2}{*}{ pH } & \multirow{2}{*}{$\begin{array}{l}\text { M.O. } \\
\text { g.dm-3 }\end{array}$} & \multirow{2}{*}{$\begin{array}{c}P \\
\mathrm{mg} / \mathrm{dm}^{3}\end{array}$} & \multicolumn{6}{|c|}{$\mathbf{m m o l} \cdot \mathbf{d m}^{-3}$} & \multirow{2}{*}{$\begin{array}{l}\mathrm{V} \\
\%\end{array}$} \\
\hline & & & & $\mathbf{H}+\mathbf{A l}$ & $\mathbf{K}$ & $\mathrm{Ca}$ & Mg & SB & CTC & \\
\hline Antes & 4,0 & 31,0 & 5,0 & 68,0 & 0,3 & 2,0 & 1,0 & 3,3 & 71,0 & 4,0 \\
\hline Após & 6,0 & 32,0 & 196,0 & 24,0 & 0,4 & 4,5 & 1,9 & 6,8 & 91,0 & 74,0 \\
\hline
\end{tabular}

${ }^{1}$ Laboratório do Departamento de Ciência do Solo (UNESP - FCA - Botucatu).

centagem de saturação de bases (Tabela 1). Para a correção do $\mathrm{pH}$ aplicou-se 2,15 g de calcário dolomítico, PRNT 90. Sem esterilizar o solo, incorporou-se: $0,1 \mathrm{~L} / \mathrm{L}$ de esterco de curral curtido, 0,9 g/L de superfosfato triplo, $0,3 \mathrm{~g} / \mathrm{L}$ de cloreto de potássio e $0,03 \mathrm{~g}$ de FRITAS $\mathrm{Br}-8 / \mathrm{L}$ de terra. 30 dias após a primeira correção foi feita uma nova análise (Tabela 1). Em cobertura utilizou-se $13,64 \mathrm{~g}$ de nitrato de cálcio, $0,48 \mathrm{~g}$ de superfosfato triplo e 2,87 g de cloreto de potássio por kilograma de solo.

As plantas foram tutoradas individualmente e conduzidas com uma haste. A irrigação por gotejamento, após o início da floração oscilou entre 2,0 e 2,5 litros/dia/vaso e na fase de produção de frutos quatro litros/dia/vaso. A colheita começou aos 10 de junho de 1996 e prolongou-se até 30 de agosto de 1996.

Foram seis tratamentos estudados, resultantes da combinação dos dois híbridos de pepino ('Nikkey' e 'Ancor 8') enxertados sobre dois híbridos de abóbora ('Ikky' e 'Tetsukabuto') além dos dois híbridos de pepino sem enxertar. Os híbridos Nikkey e Ancor 8 são amplamente utilizados pelos produtores do
Estado de São Paulo, o porta-enxerto 'Ikky' é utilizado por conferir brilho aos pepinos e 'Tetsukabuto' é tradicionalmente utilizado pela resistência a baixas temperaturas e tolerância a nematóides.

Os tratamentos foram distribuídos num delineamento de blocos ao acaso com quatro repetições e três plantas por parcela (uma planta por vaso).

Avaliou-se a altura da planta aos quinze, 45 e 120 dias, número de internódios/ planta, peso da matéria seca da raiz, número de frutos totais (colhidos com 2,5$3,5 \mathrm{~cm}$ de diâmetro e/ou 20,0-23,0 cm de comprimento), número de frutos comerciáveis $/ \mathrm{m}^{2}$ (frutos sem defeitos) e cerosidade do fruto observando-se a presença ou ausência de brilho na casca. Os resultados foram submetidos à análise da variância e as médias dos tratamentos comparadas pelo teste de Tukey a $5 \%$. Após esta análise, realizou-se o teste de correlação de Spearman entre todas as características avaliadas.

\section{RESULTADOS E DISCUSSÃO}

A análise da variância para altura da planta aos quinze e 120 dias (Tabela 2)

Tabela 2. Altura da planta em centímetros (A) aos 15, 45 e 120 dias, número de internódios/planta (I), peso da matéria seca da raiz (R), número de frutos totais (FT) e comerciáveis (FC) por metro quadrado, de plantas de pepino enxertadas e não enxertadas em abóbora ${ }^{1}$. Botucatu, UNESP-FCA , 1996. ${ }^{1}$

\begin{tabular}{|c|c|c|c|c|c|c|c|}
\hline & \multicolumn{3}{|c|}{$\mathbf{A}$} & \multirow{2}{*}{$\begin{array}{c}\text { I } \\
\text { no/pl }\end{array}$} & \multirow{2}{*}{$\begin{array}{c}\mathbf{R} \\
\mathbf{g}\end{array}$} & \multirow{2}{*}{$\begin{array}{c}\mathrm{FT}^{2} \\
\mathrm{no} / \mathrm{m} 2\end{array}$} & \multirow{2}{*}{$\begin{array}{c}\mathrm{FC}^{2} \\
\mathrm{no} / \mathrm{m} 2\end{array}$} \\
\hline & 15 & 45 & 120 & & & & \\
\hline Nikkey-Ikky & $10,5 \mathrm{bc}$ & $71,4 \mathrm{a}$ & $173,7 \mathrm{a}$ & $28,4 \mathrm{a}$ & $3,3 \mathrm{ab}$ & $48,6 \mathrm{a}$ & $29,1 \quad b$ \\
\hline Nikkey-Tetsukabuto & $10,9 \quad b$ & $64,6 \mathrm{a}$ & $162,9 \mathrm{ab}$ & $29,2 \mathrm{a}$ & $3,4 \mathrm{ab}$ & $37,4 \mathrm{~b}$ & $29,3 \quad b$ \\
\hline Ancor-Ikky & $8,1 \quad \mathrm{c}$ & $65,7 \mathrm{a}$ & $163,1 \mathrm{ab}$ & $29,6 \mathrm{a}$ & $3,6 \mathrm{a}$ & $52,9 \mathrm{a}$ & $40,3 \mathrm{a}$ \\
\hline Ancor-Tetsukabuto & $10,5 \mathrm{bc}$ & $65,8 \mathrm{a}$ & $154,7 \mathrm{abc}$ & $28,8 \mathrm{a}$ & $3,0 \mathrm{ab}$ & $46,0 \mathrm{ab}$ & $32,4 \mathrm{ab}$ \\
\hline Nikkey & $14,4 \mathrm{a}$ & $65,8 \mathrm{a}$ & $124,0 \quad \mathrm{c}$ & $23,2 \mathrm{~b}$ & $2,3 \quad b$ & $44,3 \mathrm{ab}$ & $23,6 \quad b$ \\
\hline Ancor & $14,6 \mathrm{a}$ & $62,4 \mathrm{a}$ & $127,2 \mathrm{bc}$ & $26,2 \mathrm{ab}$ & $2,9 \mathrm{ab}$ & $43,4 \mathrm{ab}$ & $25,0 \quad \mathrm{~b}$ \\
\hline Média & 11,5 & 66,0 & 150,9 & 27,6 & 3,1 & 45,5 & 30,0 \\
\hline Tukey (5\%) & 2,7 & 18,3 & 37,6 & 4,4 & 1,1 & 11,0 & 9,3 \\
\hline $\mathrm{CV}(\%)$ & 9,8 & 11,6 & 10,4 & 6,7 & 15,53 & 10,1 & 13,0 \\
\hline
\end{tabular}

*/ Colunas seguidas pelas mesmas letras não diferem entre si pelo teste de Tukey à $5 \%$.

1/ Dados originais (média de 3 plantas e 4 repetições).

$2 /$ Considerando espaçamento de $0,5 \times 0,7 \mathrm{~m}$. 
detectou diferenças significativas entre os tratamentos e nenhuma diferença aos 45 dias. O teste de Tukey (5\%) encontrou que aos quinze dias as plantas não enxertadas cresceram mais, sendo que, os tratamentos 'Nikkey' e 'Ancor' atingiram $14,4 \mathrm{~cm}$ e $14,6 \mathrm{~cm}$, respectivamente, enquanto as enxertadas demonstraram menor altura, com o tratamento 'Ancor-Ikky' apresentando 8,1 cm. Aos 45 dias os seis tratamentos mostraram valores próximos e, aos 120 dias, as plantas enxertadas foram mais altas, destacando-se o tratamento 'NikkeyIkky' com 173,7 cm em oposição ao tratamento 'Nikkey' com 124,0 cm.

As diferenças entre os tratamentos para o número de internódios/planta aos 120 dias foram significativas (Tabela 2). $\mathrm{O}$ tratamento que mais internódios apresentou foi 'Ancor-Ikky' com a média de 26,6 internódios/planta, enquanto o híbrido Nikkey sem enxertia apresentou a média de 23,2 internódios/planta. Não houve diferenças estatísticas entre as plantas enxertadas. Houve correlação entre número de internódios com altura da planta aos 120 dias $(r=0,86)$, indicando que a enxertia aumentou a altura $\mathrm{e}$ número de internódios/planta.

O desenvolvimento do sistema radicular foi melhor no tratamento 'Ancor-Ikky', sendo que a média do peso da matéria seca da raiz foi de 3,6 g, em oposição ao tratamento 'Nikkey' que registrou 2,3 g. Embora sem diferença estatística entre os tratamentos restantes, o peso da matéria seca da raiz foi ligeiramente superior nas abóboras quando comparadas com o pepino (Tabela 2).

A parte aérea das plantas pode ter sido influenciada pelo vigor do sistema radicular das abóboras 'Ikky' e 'Tetsukabuto', aumentando a altura de 'Nikkey' e 'Ancor', corroborando com as afirmações de Yamakawa (1982) quanto aos porta-enxertos selecionados que incrementaram a eficiência da cultura e conferiram vigor à planta. $\mathrm{O}$ vigor das raízes da abóbora pode ter sido eficientemente aproveitado nos meses de junho e julho, onde se registraram temperaturas médias mínimas de 10 e $12^{\circ} \mathrm{C}$, respectivamente, condição climática prejudicial para o desenvolvimento do pepino, já que a enxertia sobre materiais vigorosos é recomendada para suportar períodos curtos de temperaturas baixas, devido a que as raízes da abóbora continuam absorvendo os nutrientes e a água nestas condições (Liebig, 1985).

A análise da variância do número de frutos totais e comerciáveis identificou diferenças estatísticas (Tabela 2). O teste de Tukey (5\%) demonstrou que 'Nikkey' e 'Ancor' enxertados sobre 'Ikky' produziram mais frutos, 48,6 e 52,9 frutos totais $/ \mathrm{m}^{2}$, respectivamente. A produção de frutos de pepinos enxertados pode ser influenciada pelo aumento de flores femininas por causa de alguma alteração nos reguladores da expressão sexual do porta-enxerto (Takahashi et al., 1982 e Friedlander et al., 1977).

O porta-enxerto 'Ikky' incrementou a produção de frutos de 'Nikkey' em 9,7\% e de 'Ancor' em 21,9\%. O portaenxerto 'Tetsukabuto' aumentou a de 'Ancor' em 5,4\% e diminuiu a de 'Nikkey' em 15,6\%. A menor produção $\left(37,4\right.$ frutos totais $\left./ \mathrm{m}^{2}\right)$ foi representada pelo tratamento 'Nikkey-Tetsukabuto', discordando com o desenvolvimento da planta respaldado pelos valores da altura e do número de internódios atingidos pelo mesmo tratamento. Este fato foi explicado por Kawaide (1985) que afirmou que existem híbridos usados como porta-enxertos que podem passar muito vigor à planta enxertada, e com isso excessiva proliferação de folhas. É muito provável que 'Tetsukabuto' tenha induzido muito vigor a 'Nikkey' acusando assim proliferação de folhas, resultando em menor produção de frutos o que está em concordância com o observado neste experimento. O tratamento 'Ancor-Ikky' produziu 40,3 frutos comerciáveis $/ \mathrm{m}^{2}$, sendo seguido pelo tratamento 'AncorTetsukabuto' com 32,4 frutos comerciáveis $/ \mathrm{m}^{2}$, demonstrando que neste experimento, a maior eficiência foi obtida pelo 'Ancor' enxertado, embora o teste de Tukey a 5\% não tenha detectado diferenças entre os demais tratamentos. Os híbridos não enxertados, 'Nikkey' e 'Tetsukabuto' atingiram menores rendimentos, 23,6 e 25,0 frutos comerciáveis $/ \mathrm{m}^{2}$, respectivamente. A produção de frutos comerciáveis nas plantas não enxertadas foi $25,8 \%$ menor que as enxertadas, sendo considerados comerciáveis, 59,8\% da produção de frutos totais do tratamento 'Nikkey-Ikky'; 78,4\% de 'Nikkey-Tetsukabuto'; 76,1\% de 'Ancor-Ikky'; 70,5\% de 'AncorTetsukabuto'; 53,2\% de 'Nikkey' e $57,8 \%$ de 'Ancor'. O porta-enxerto 'Ikky' aumentou em 23,2\% o número de frutos comerciáveis $/ \mathrm{m}^{2}$ em 'Nikkey' e $61,0 \%$ em 'Ancor', enquanto, o portaenxerto 'Tetsukabuto' aumentou em $24,2 \%$ a produção de frutos comerciáveis em 'Nikkey' e em 29,6\% em 'Ancor'. Estes resultados sugerem que a enxertia influencia positivamente a qualidade visual dos frutos, concordando com o relato de Kawaide (1985), de que a enxertia em cucurbitáceas, usando cavalos selecionados, incrementa a eficiência em rendimento diminuindo a porcentagem de frutos com distúrbios fisiológicos. Isso pode ser explicado pela maior capacidade de absorção de nutrientes pelas plantas enxertadas durante a época mais fria (Liebig, 1985; Oda, 1995).

Também foi possível observar que os frutos das plantas enxertadas sobre 'Ikky' apresentavam brilho na casca concordando com Kawaide (1985), e presença de cerosidade nos frutos das plantas não enxertadas e enxertadas sobre 'Tetsukabuto'.

\section{AGRADECIMENTOS}

À Fundação de Amparo à Pesquisa do Estado de São Paulo (FAPESP), aos Profs. Drs. Júlio Nakagawa e Roberto Lyra Villas Boas.

\section{LITERATURA CITADA}

CARVALHO W.A.; ESPÍNDOLA, C.R.; PACCOLA, A.A. Levantamento de solos da Fazenda Lageado - Estação Experimental "Presidente Medina". Boletim Cientifico Faculdade Ciências Agronômicas, UNESP, Botucatu, n. 1, p. 1 - 95, 1983.

FRIEDLANDER, D.; ATSMON, D.; GALUN, E. The effect of grafting on sex expression in cucumber. Plant Soil, v. 18, p. 1343 - 50, 1977.

JANICK, J. A ciência da horticultura. Rio de Janeiro: Freitas Bastos, 1966. p. 224 - 329.

JANOWSKI, G.; SKAPSKI, H. Hydro-peat method for greenhouse cucumber production. Horticultural Abstract, v. 56, p. 253, 1985. Abstract 02424.

KAWAIDE, T. Utilization of rootstocks in cucurbits production in Japan. Japan Agricultural Research Quartely, v. 18, n. 4, p. $285-8,1985$. 
CAÑIZARES, K.A.L.; GOTO, R. Crescimento e produção de pepino em função da enxertia.

LIEBIG, H.P. Model of cucumber growth and yield. I. Raising the crop under low temperature regimes. Acta Horticulturae Sinica, v. 156, p. 127 - 37, 1985.

ODA, M. New grafting methods for fruit-bearing vegetables in Japan. Japan Agricultural Research Quartely, v. 29, p. 187 - 94, 1995.
ODA, M.; TSUJI, K.; SASAKI, H. Effect of hypocotyl morphology on survival rate and grow of cucumber seedlings grafted on Cucurbita spp. Japan Agricultural Research Quartely, v. 26, p. 259 - 63, 1993.

SILVA JUNIOR, A.A.; SOPRANO, E.; VIZZOTTO, V.J.; MACEDO, S.G. Caracterização de deficiências nutricionais em pepineiro. Santa Catarina: EPAGRI, 1995. 35 p.
TAKAHASHI, H.; SAITO, T.; SUGE, H. Intergeneric translocation of floral stimulus across a graft in monoecious cucurbitaceae with special reference to the sex expression of flowers. Plant Soil, v. 23, n. 1, p. 1 - 9, 1982.

YAMAKAWA, K. Use of rootstocks in solanaceous fruit vegetable production in Japan. Japan Agricultural Research Quartely, v. 15, n. 3, p. 175 - 79, 1982.

CARVALHO, C. G. P. de; OLIVEIRA, V. R.; CASALI, V. W. D.; CRUZ, C. D. Correlações canônicas entre componentes primários e secundários da produção de frutos em pimentão. Horticultura Brasileira, Brasília, v. 16, n. 2, p. 113 - 118, novembro 1998.

\title{
Correlações canônicas entre componentes primários e secundários da produção de frutos em pimentão.
}

\author{
Claudio G. P. de Carvalho' ${ }^{1}$ Valter R. Oliveira²; Vicente W. D. Casali ${ }^{3}$; Cosme D. Cruz ${ }^{1}$. \\ ${ }^{1}$ UFV - Dep ${ }^{\text {to }}$ Biologia Geral, 36571-000 Viçosa - MG; ${ }^{2}$ EPAMIG - Centro Tecnológico do Centro Oeste, C. Postal 35701-970 Sete \\ Lagoas - MG; ${ }^{3}$ UFV - Dep ${ }^{\text {to. }}$ Fitotecnia, 36571-000 Viçosa - MG.
}

\section{RESUMO}

Avaliaram-se as associações existentes entre componentes primários e secundários da produção de frutos, em genótipos de pimentão, por meio da análise de correlações canônicas. Além disso, realizou-se o estudo da adequação dos dados a esta análise em decorrência da existência de multicolinearidade expressa nas matrizes de correlações utilizadas. O ensaio foi conduzido no município de Viçosa (MG), no período de 6 de outubro de 1993 a 19 de março do 1994. A umidade relativa do ar média anual da região é de $80 \%$, a temperatura média anual é de $20^{\circ} \mathrm{C}$ e a precipitação média anual é de $1340 \mathrm{~mm}$. O delineamento experimental utilizado foi blocos completos casualizados, com três repetições. Cada parcela foi composta de uma fileira com seis plantas, espaçadas $0,40 \mathrm{~m}$, mantendo-se um espaçamento entre linhas de $0,9 \mathrm{~m}$. As quatro plantas centrais constituíram a parcela útil. $O$ estudo das correlações e dos pares canônicos revelou que há dependência significativa a $1 \%$ de probabilidade, pelo teste de qui-quadrado, entre caracteres morfológicos e agronômicos (diâmetros do pendúnculo e do caule e alturas da planta e da primeira bifurcação) e de fruto (comprimento, largura, relação comprimento/largura do fruto e espessura da polpa) com os componentes primários da produção (peso médio e número total de frutos). A associação mais expressiva foi estabelecida entre estes componentes e caracteres do fruto. Para estes caracteres, deve-se considerar simultaneamente o comprimento e a largura do fruto e a espessura da polpa para se obter ganhos em número total e, principalmente, em peso médio de frutos. Entre os caracteres morfológicos e agronômicos, diâmetro do pedúnculo apresentou importância mais destacada e poderá permitir a obtenção de resposta correlacionada com peso médio de frutos.

Palavras-chave: Capsicum annuum L., multicolinearidade, peso médio de frutos, características de frutos.
ABSTRACT

Canonical correlations between primary and secondary components of fruit production in sweet-pepper.

Associations between primary and secondary components of fruit production in sweet-pepper genotypes were evaluated by means of canonical correlation analysis. Additionally, a study of data fitness for this analysis was done as a result of observing multicollinearity in the correlation matrices used. The experiment was carried out in the municipality of Viçosa (MG), during the period from October $6^{\text {th }} 1993$ to March $19^{\text {th }} 1994$. The mean annual relative air humidity of the region is $80 \%$, the mean annual temperature is $20^{\circ} \mathrm{C}$ and the mean annual precipitation is $1340 \mathrm{~mm}$. The experimental design used was complete randomized blocks with three replications. Each plot had a row with six plants, spaced $0.40 \mathrm{~m}$, with a space of $0.9 \mathrm{~m}$ between lines. The four central plants represented the useful plot. The study of correlations and of canonic pairs revealed that there was a significant dependence $(\mathrm{P}>$ $0.01)$, by the chi-square test, between morphological and agronomic characters (peduncle and stem diameters, and plant and first bifurcation heights) and fruit (fruit length, width and length/width relation and pulp thickness) with the primary yield components (fruit average weight and total number). The most expressive association between these components was established with fruit characters. For these characters, fruit length, width and pulp thickness should be considered simultaneously to obtain gains in total number of fruits and, mainly, in fruit average weight. For morphological and agronomic characters, peduncle diameter stands out as it one for morphological and agronomic characters, peduncle diameter stands out as it is the one character correlated with fruit average weight, correlated with fruit average weight.

Keywords: Capsicum annuum L., multicollinearity, fruit average weight, fruit characters.

(Aceito para publicação em 18 de setembro de 1998) 